\title{
Stress Estimation of Beam Structures Based on 3D Coordinate Information from Terrestrial Laser Scanning
}

\author{
Hong Min Lee \\ Industrial Engineering Department \\ Samsung Engineering Co. \\ Seoul, Korea \\ e-mail: hm01.1ee@samsung.com
}

\author{
Hyo Seon Park \\ Dept. of Architectural Engineering \\ Yonsei University \\ Seoul, Korea \\ e-mail: hspark@yonsei.ac.kr
}

\begin{abstract}
Terrestrial laser scanner can remotely obtain 3D coordinate data of a structural member without installation of sensors or reflective targets on the structure. In this paper, to overcome the limitation of the conventional measuring system based on strain sensors, relative changes in 3D coordinate data obtained from a laser scanner are used for estimation of the maximum stress in beam structures in a building. The estimated stress from 3D image data was compared with the maximum stress measured directly from electrical strain gages.
\end{abstract}

Keywords-structural health monitoring; structural safety; steel beam; maximum stress

\section{INTRODUCTION}

To rationally control the safe ty and serviceability of structural members subjected to both design loads and unexpected overloads simultaneously, periodic monitoring of stress and displacement in a m ember is required. For this reason, various te chniques for measurement of structural responses have been developed [1-15].

However, most strain sensors used in measurement of strains in a member can cover a limited range of structural members. There is a limitation on assessment of the safety of a str uctural member using strain sensors attached at fixed locations. In this paper, stress estimation model using a $3 \mathrm{D}$ laser scanner terrestrial is presented for monitoring of the safety and serviceab ility of beam structures. In addition to estimation of maximum stress, the distribution of stresses in a structural member is visualized by a finite element method (FEM). The use of $3 \mathrm{D}$ laser scanner makes it possible to evaluate the safety of a beam structures without installation process of strain sensors.

The proposed model was tested by com paring the maximum stress from 3D laser scan ner with the maximum stress from strain sensors install ed on the surface of the beam structure.

\section{3D COORDINATE INFORMATION}

A laser scanner system uses laser signal to obtai $\mathrm{n}$ three-dimensional coordinate data. $[16,17]$. A 1 aser scanner obtains 3D coordinates of an object by measuring the time required for the laser pulse to travel.

Figure 1 shows a scanned image of steel beam using $3 \mathrm{D}$ coordinates from a 1 aser scanner. In this paper, the error ranges for $3 \mathrm{D}$ coordinate data from a laser scanner located $100 \mathrm{~m}$ from a target to be monitored in horizontal and vertical directio ns are about 10 and $7 \mathrm{~mm}$, respectively

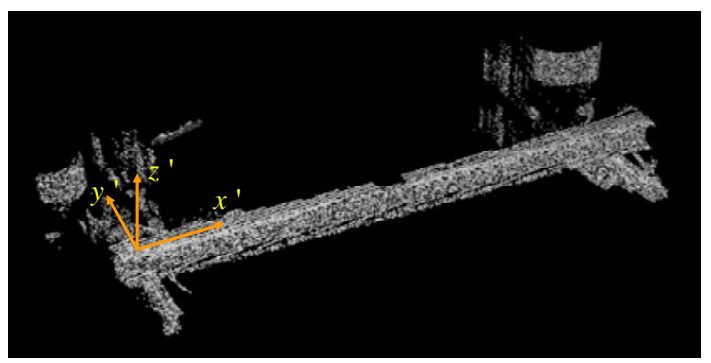

FIGURE 1. SCANNED IMAGE OF A STEEL BEAM BASED ON 3D COORDINATE DATA FROM A LASER SCANNER.

\section{STRESS ESTIMATION MODEL}

The basic principle of the method for estimating the maximum strain of structures is to minimize errors in measurements of displacements using the least square method from the 3D coordinates obtained from the scanner with errors in vertical and ho rizontal directions, as shown in Fig. 1.

For estimation of the $m$ aximum stress based on deformed shape from the 3D coordinates of a s tructure from a laser scanner [3,9], the deflection of the steel beam structure subjected to vertical loads at an arbitrary horizontal location can be e xpressed in a $n$th order polynomial in Eq. (1).

$$
z(x)=c_{0} x^{n}+c_{1} x^{n-1}+\cdots+c_{n-2} x^{2}+c_{n-1} x^{1}+c_{n}
$$

where $c_{0}, c_{1}, \cdots, c_{n-2}, c_{n-1}, c_{n}$ represent the coefficients of the polynomial functions to be determined using three dimensional coordinate data obtained from a laser scanner 
and the least square method. For an optimal degree of polynomial, the coef ficients $c_{0}, c_{1}, \cdots, c_{n-2}, c_{n-1}, c_{n}$ for generation of the equation for a deformed shape of a steel beam. After minimization of errors in estimation of vertical deflection of a steel beam, a general equat ion of the deformed shape of steel beam is used to estimate the vertical deflection, $z(x)$, at an arbitrary location of a structure.

For estimation of the stress of a structure based on the estimated deflections, a FEM is used for analyzing the stress distribution using the optimized deformed shape in Eq. (1).

\section{ApPlication to Stress Estimation OF STEEL BEAMS}

To test the performance of the stress estimation model using a laser scanner, the three point bending test was performed for the steel b eam. As shown in Fig. 2, the experimental specimen is simply supported steel beam of H- $100 \times 100 \times 6 \times 6$ with the yield strength of $F_{y}=253.3$ $\mathrm{MPa}$.

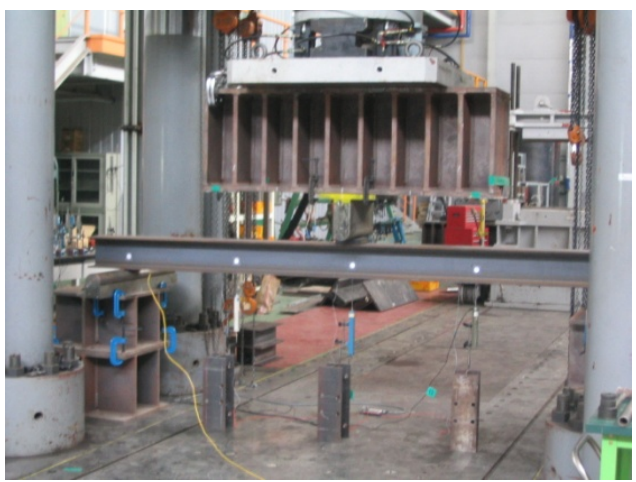

FIGURE 2. EXPERIMENTAL SETUP FOR THREE POINT BENDING TEST OF SIMPLY SUPPORTED STEEL BEAM SUBJECTED TO A POINT LOAD.

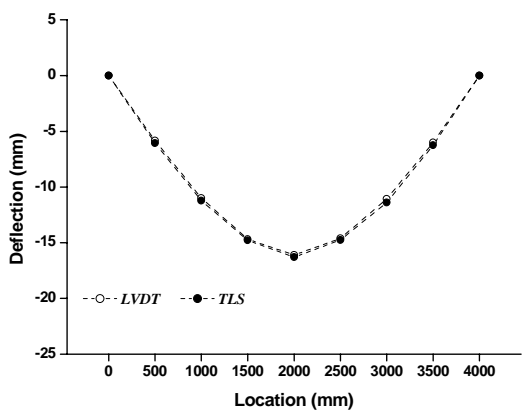

FIGURE 3. ESTIMATED AND MEASURED DEFORMED SHAPES THE STEEL BEAM.

In the mid-point of the steel beam, a $9.66 \mathrm{kN}$ of concentrated load, which is equivalent to approximately $1 / 2$ of yield load of beam, was applied. For the comparison of deflections, seven line ar variable displacement transducers (LVDTs) and electrical strain gauges (ESG) are installed at $1 / 8,1 / 4,31 / 8,1 / 2,51 / 8,31 / 4$, and $71 / 8$ positions of the steel beam.

For estimation of the deformed shape, approximately 40,000 and 20,000 data were obtained from the surfaces of web and upper flange of steel beam, respectively. Based on the scattered data about 3D coordinates, in Fig. 3 , the de formed shape was es timated and compared with measured deformed shape. This in dicates that the deformed shape of steel beam was estimated at an approximate accuracy of smaller than $0.5 \mathrm{~mm}$ at all locations.

To investigate the effectiveness in esti mating the stress of the steel beam, the beam structure was divided into 2 elements, 4 elements and 8 ele ments. Data about the deformed shape which was esti mated were en tered into the program for interpreting FEM.

In pursuit of the methods for assessing the overall health of beam structure, rather than the measurement of structural response at specific location of beam structure, the stress of beam structure was calculated using the data about the displacement which was obtained at locations of $1 / 2$ ( 2 elements), at locations of $1 / 4,1 / 2,31 / 4$ (4 elements), and at 1 ocations of $1 / 8,1 / 4,31 / 8,1 / 2,51 / 8,31 / 4,71 / 8(8$ elements).

As shown in Fig. 4, the distributions of stresses in the beam along the length of the beam can automatically be estimated. According to FEM for assessing the stress, the maximum stress error was $5.64 \mathrm{MPa}$ (a location of $1 / 8$ ) following the use of 2 elements, $7.10 \mathrm{MPa}$ (a location of $1 / 4)$

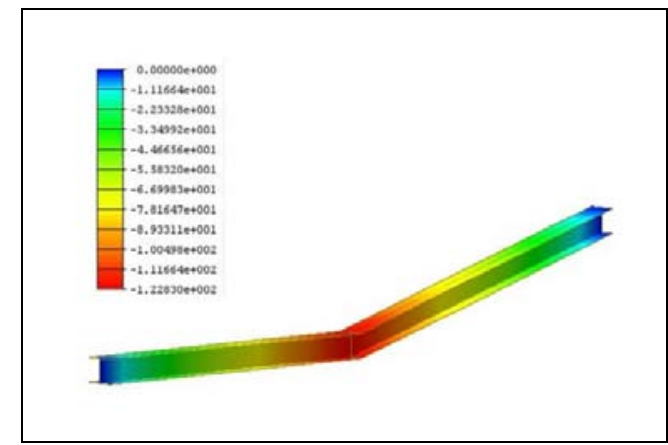

(a) 2 elements

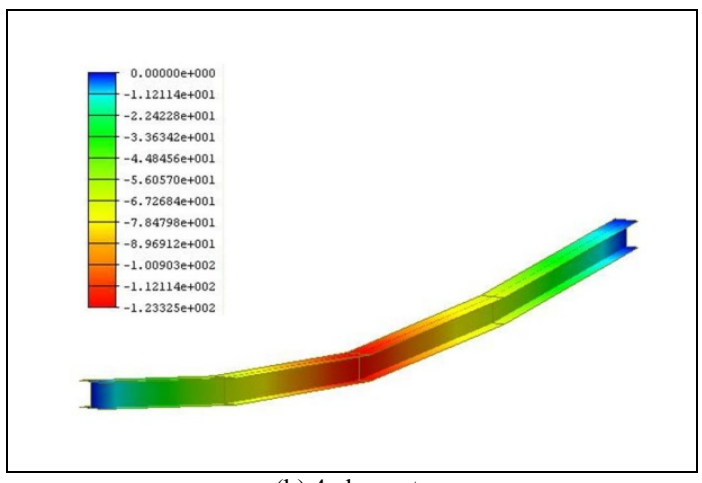

(b) 4 elements 


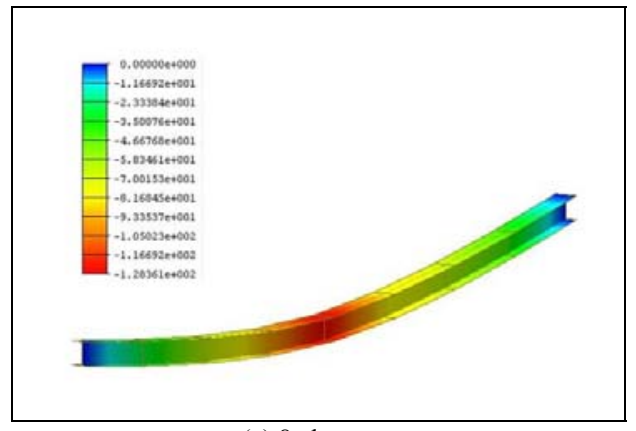

(c) 8 elements

FIGURE 4. STRESS DIAGRAM AUTOMATICALLY ESTIMATED FROM FEM USING THE DEFORMED SHAPES.

following the use of 4 elements and $11.84 \mathrm{MPa}$ (a location of 51/8) following the use of 8 e lements. The maximum stress error was $\mathrm{n}$ oted to be $11.84 \mathrm{MPa}$ (approximately $5 \%$ based o $\mathrm{n} \mathrm{F}_{\mathrm{y}}=253.3 \mathrm{MPa}$ ) on $\mathrm{t}$ he stiffness methods.

It was confirmed that the deformed shape of structure can be estimated with a laser scanner s ystem and the stress of beam structure can be estimated based on the estimated deformed shape. The stress of steel beam was calculated using the data about the displacement at several locations in the direction of the length of beam structure. The more the number of elements is increased, it was clear that the err or in estimation of the stress could be increased because the displacement error is more sensitive. In cases in which the deformed shape of a beam cannot be predicted, however, it could be expected that the data about displacement at specific location according to this is mandatory.

\section{CONCLUSIONS}

The measured structural responses of buildings and infrastructures are different from the calculated structural responses based on struct ural analysis due to the uncertainties in various as sumptions used in s tructural modeling. Furthermore, the structural performance of building structures can be changed due to unexpected use of building causing changes in design load and load path.

For this reason, in these days, various researches have been reported on development of monitoring of structural responses.

A laser scanner system is the system that can remotely obtain three dimensional coordinates of a structure using laser. Use of the 1 aser scanner in measurements of changes in 3D coordinates of a structure allows estimation of 3D coordinate data for not on ly the specific point but also whole structure without attachment of reflective markers.

In this paper, to overcome the limitation of the conventional measuring system based on strain senso rs, relative changes in 3D co ordinate data o btained from a laser scanner are used for estimation of the maximum stress in beam structures in a building.

The performance of the model is verified through three point bending test of $4 \mathrm{~m}$ long steel beam. It is verified that the $u$ se of laser scanner $w$ as effective in estimating the stress of structure.

\section{ACKNOWLEDGMENT}

This work was sup ported by the National Research Foundation of Korea (NRF) grant funded by the Korea government (MSIP) (No. 2011-0018360).

\section{REFERENCES}

[1] H. S. Park, S. M. Jung, H. M. Lee, Y. H. Kwon, and J, H. Seo (2007), "Analytical models for assessment of the safety of multispan steel beams based on average strains from long gauge optic sensors", Sensors and Actuators A, vol. 137, pp. 6-12, 2007.

[2] S. Bhalla, Y. W. Yang, J. Zhao, C. K. Soh, "Structural health monitoring of underground facilities - Technological issues and challenges", Tunnelling and underground space technologies, vol. 20, pp. 487-500, 2005.

[3] H. M. Lee and H. S. Park, "Gage-free stress estimation of a beamlike structure based on terrestrial laser scanning", Computer-Aided Civil and Infrastructure Engineering, vol. 26, pp. 647-658, 2011.

[4] J. R. Casas and J. S. Cruz, "Fiber optic sensors for bridge monitoring" Journal of bridge engineering, ASCE, vol. 8, pp. 362373, 2003.

[5] D. Inaudi, S. Vu rpillot, N. Casanova, and P. K ronenberg, "Structural monitoring by curvature analysis using interferometric fiber optic sensors" Smart Materials and Structures, vol. 7, pp. 199-208, 1998.

[6] H. S. Park, H. M. Lee, H. Adeli, and I. Lee, "A new approach for structural health monitoring of structures: terrestrial laser scanning”, Computer-Aided Civil and Infrastructure Engineering, vol. 22, pp. 19-30, 2007.

[7] M. Pasquale, "Mechanical sensors and a ctuators" Sensors and actuators A: Physical, vol. 106, pp. 142-148, 2003.

[8] H. S. Park, H. S. Jung, Y. H. Kw on, and J. H. Seo, "Mathematicl models for assessment of the safety of steel beams based on average strains from long gage optic sensors", Sensors and actuators A: Physical, vol. 125, pp. 109-113, 2006.

[9] H. M. Lee and H. S. Park, "Esimation of deformed shapes of beam structures using 3D coordinate information from terrestrial laser scanning", Computer Modelling in Engineering \& Sciences, vol. 29, pp. 29-44, 2008

[10] C. K. Y. Leung, "Fiber optic sensors in concrete: the future?" NDT\&E International, vol. 34, pp. 85-94, 2001.

[11] R. C. Tennyson, A. A. Mufti, S. Rizkalla, G. Tadros, and B Benmokrane, "Structural health $\mathrm{m}$ onitoring of innovative bridges in Canada with fiber optic sensors" Smart Materials and Structures, vol. 10, pp. 560-573, 2001.

[12] H. M. L ee, J. M. Kim, K. Sho, and H. S. Park, "A wireless vibrating wire sensr node for continuous structural health monitoring", Smart Materials and Sructures, vol. 19, 055004, 2010.

[13] S. W. Choi, B. R. Kim, H. M. Lee, Y. Kim, and H. S. Park, "A deformed shape monitoring model for buildng structures base on a 2D laser scanner", Sensors, vol. 1, pp. 6746-6758, 2013

[14] H. S. Park, S. Son, S. W. Choi, and Y. Kim, "Wireless laser range finder system for vertical displacement monitoring of mega-truss during construction”, Sensors, vol. 13, pp. 5796-5813, 2013.

[15] S. W. Choi, J. Lee, J. M. Kim, and H. S. Park, "Design and application of a field sesning system for ground anchors in slopes", Sensors, vol. 13, 3739-3752, 2013.

[16] Optech, "Optech ILRIS-3D Scanner Brochure", from: http://www.optech.ca/, 2012.

Trimble, "Trimble GX 3 D Scanner Brochure", from: http://www.trimble.com/, 2012. 